\title{
New Measurement Method for Direct Cooled Power Electronic with Transmission Fluids
}

\author{
Andreas Griesinger ${ }^{1}$, Christian Windel ${ }^{2}$, Yungwan Kwak ${ }^{3}$, Arthur Petuchow ${ }^{4}$ \\ ${ }^{1}$ Zentrum für Wärmemanagement, Stuttgart, Germany \\ ${ }^{2}$ Mercedes-Benz AG, Development Passanger Cars, Stuttgart, Germany \\ ${ }^{3}$ Afton Chemical Corporation, Richmond, USA \\ ${ }^{4}$ Afton Chemical GmbH, Hamburg, Germany \\ Email: andreas.griesinger@zfw-stuttgart.de
}

How to cite this paper: Griesinger, A., Windel, C., Kwak, Y. and Petuchow, A. (2021) New Measurement Method for Direct Cooled Power Electronic with Transmission Fluids. Journal of Electronics Cooling and Thermal Control, 10, 1-13.

https://doi.org/10.4236/jectc.2021.101001

Received: March 1, 2021

Accepted: March 28, 2021

Published: March 31, 2021

Copyright (c) 2021 by author(s) and Scientific Research Publishing Inc. This work is licensed under the Creative Commons Attribution International License (CC BY 4.0).

http://creativecommons.org/licenses/by/4.0/ Open Access

\begin{abstract}
Power Electronic (PE) will play an essential role in future drive concepts. Nowadays, mainly water/glycol-based cooling media are used to cool PE. Due to their high electrical conductivity (EC), water/glycol-based coolants cannot be used for direct cooling of the electrical components. Direct cooling concepts with dedicated transmission fluids show potential usage of fluid in direct contact with electrified parts. This results in special requirements for the fluids and materials. The aimed action as a coolant requires a defined measurement and characterization of fluid properties and heat transfer in order to assess the cooling ability of a fluid. The purpose of the work was to develop a new measurement setup based on the thermal transient method with which the thermal requirements of cooling fluids for a direct cooling concept can be assessed. With this method, relevant transmission fluids have been tested and the thermal performance compared to indirect cooling effect of water/glycol is discussed. The result of the work is that the measurement method is very well suited for the application-related evaluation of the fluids. Direct oil cooling with transmission fluids could increase heat transfer coefficient by a factor of 3 to 8 , compared to the indirect cooing with water/glycol as cooling media.
\end{abstract}

\section{Keywords}

Thermal Transient Method, Heat Transfer Coefficient, Direct Cooling, Transmission Fluids

\section{Introduction}

The trend in the automotive industry towards electrified powertrain is continuously increasing. Power Electronic (PE) is one of the main components in an 
electrified powertrain in both hybrid and purely electric driven cars. Several ongoing research projects including projects done directly at OEMs show the potential of PE in terms of increasing efficiency in electrified drive trains. The majority of them have the goal of converting high electrical energy in order to achieve the optimal performance of electric machines. One side effect of this goal is the continuously increased heat input, which explains why thermal management becomes more and more important. Especially at starting conditions, relatively high current flows through semiconductors and results in thermal stress which can lead to welding of switch contacts. Direct cooling of the included semiconductors on a PE would reduce the thermal stress and optimize heat transfer.

The state of the art for cooling of PE is the usage of water/glycol-based cooling media. Due to their high electrical conductivity (EC), water/glycol-based coolants cannot be used for direct cooling of the electrical components and therefore is limited in the optimization of heat transfer.

Direct cooling concepts, meanwhile known for their usage in electric motors, with dedicated transmission fluids show potential usage of fluid in direct contact with electrified parts e.g. coils. Hence, there is a high interest to apply direct cooling and use a single fluid for PE, transmission, and e-motor. A resulting reduction of the water/glycol cooling circuit and saving of associated periphery offers further options to increase efficiency.

Direct cooling of PE with an electrified transmission fluid means to fulfill combined requirements. There are conventional requirements defined by the transmission design but also additional requirements due to new materials (compatibility) and conditions (electrified environment). The aimed action as a coolant requires a defined measurement and characterization of fluid properties in order to assess the cooling ability of a fluid.

More often than not, a clever heat sink design or surface for direct oil cooling can increase the heat transfer area and heat transfer coefficient without elevating the pressure drop of the flow.

Conventional methods of determining heat transfer coefficients use thermocouples, which measure the temperatures as a result of an imposed heat flux. Particularly, in the case of inhomogeneous temperature distributions, there is the problem of correctly assessing the temperature distribution and weighting the heat transferring surfaces.

The new measuring method is intended to measure the actual thermal resistance between the heat sink surface and the fluid. From this, if the effective heat transferring area is known, the heat transfer coefficient can be calculated.

With the new method, relevant transmission fluids have been tested and the thermal performance compared to indirect cooling effect of water/glycol is discussed.

\section{Measurement Setup}

For the evaluation of a cooling circuit the rate of heat transfer and the pressure 
drop are the most important parameters which describe the performance of the setup.

While measuring the pressure drop is often a standard measurement task (depending on exact accuracy requirements), the transferred heat flow is difficult to measure. In principle, the mass flow $\dot{m}$ in $\mathrm{kg} / \mathrm{s}$, the specific heat $c$ in $\mathrm{J} /(\mathrm{kg} \cdot \mathrm{K})$, and the inlet and outlet fluid temperature $T_{\text {in }}$ and $T_{\text {out }}$ of the heat sink result in the transferred heat flow:

$$
\dot{Q}=\dot{m} c\left(T_{\text {in }}-T_{\text {out }}\right)
$$

The difficulty with the practical measurement is that the temperature difference $T_{\text {in }}-T_{\text {out }}$ is only a few Kelvin. Standard temperature measurement technology is insufficient to achieve suitable accuracy. In addition, the temperatures at the fluid inlet and outlet must be homogeneous across the cross-section, which is difficult to achieve in practice.

A new measurement system has been developed for the accurate characterization of liquid cooling for power electronics at ZFW Stuttgart, which is based on the thermal transient method [1] [2]. The junction of an Insulated-Gate Bipolar Transistor (IGBT) module is heated by a step function, Figure 1 (top left). The thermal response to the step function, the temperature rise of the junction, is detected by a temperature sensitive parameter of the semiconductor such as the forward voltage of a diode. The temperature is obtained by the calibration of the sensitive parameter in a thermostat or on a cool plate before the measurement. The measured temperature rise normalized with the heating power $P$, results in the $Z_{\text {th }}$-Curve (Figure 1, top right). The three curves in the diagram represent different mass flows (here $5 \mathrm{~kg} / \mathrm{min}, 10 \mathrm{~kg} / \mathrm{min}$, and $15 \mathrm{~kg} / \mathrm{min}$ ) in the heat sink of the PE.

The $\mathrm{Z}_{\mathrm{th}}$-curve as step response includes all relevant information on the thermal path, from the heat source (junction of IBGT module) to the heat sink (coolant fluid). In concrete terms, this means the thermal resistances in $\mathrm{K} / \mathrm{W}$ and heat capacities in $\mathrm{J} / \mathrm{K}$ of the individual layers in the heat path. These can be determined accordingly by mapping the heat path using a basic RC thermal model (Cauer thermal model). The thermal resistances and thermal capacities of the individual layers are calculated from the $\mathrm{Z}_{\mathrm{th}}$-curve by running mathematical conversions [1]. The result of this mathematic conversion is the so-called structure function, Figure 1 (bottom). The projection on the corresponding functional section on the $\mathrm{R}_{\mathrm{th}}$-axis provides the thermal resistance $\mathrm{R}_{\mathrm{th}}$ of the corresponding layer in the heat path, and the projection on the $\mathrm{C}$-axis, the corresponding heat capacity.

Although the heat transfer in the power semiconductor is relevant, the thermal resistance $R_{t h, h}$ between the surface of the heat sink and the cooling medium is of particular interest. $R_{t h, h}=1 /(h \cdot A)$ applies, with the heat transfer coefficient $\mathrm{h}$ in $\mathrm{W} /\left(\mathrm{m}^{2} \cdot \mathrm{K}\right)$ and the heat transfer area $A$ of the heat sink. The heat transfer coefficient $h$ is a function of the material properties of the coolant, geometric conditions, flow velocity, and flow type. As the velocity and turbulence of the flow 
rise, $h$ increases and the thermal resistance between the surfaces of the heat sink and coolant decreases [3].

The part marked blue of the structure functions (Figure 1, bottom) stands for the mass flows of $5 \mathrm{~kg} / \mathrm{min}, 10 \mathrm{~kg} / \mathrm{min}$ and $15 \mathrm{~kg} / \mathrm{min}$. With decreasing mass flow, $h$ is becoming smaller and $R_{t h, h}$ increases. The separation point marks the surface of the heatsink, when the applied heat is leaving the solid surface and passes into the liquid. To the left of this point, the curves are congruent because this part represents the inner structure of the module. To the right, the curves are different because of the different mass flows. In addition to the measurement of $h$, the mass flow, pressure conditions, and temperatures of the flow are recorded. The measurement uncertainty for these values obtained is typically well below 0.5 percent. For the mass flow, a Coriolis flow meter is used. The advantage is that the mass flow is measured directly and no conversion from volume flow or flow velocity is necessary.
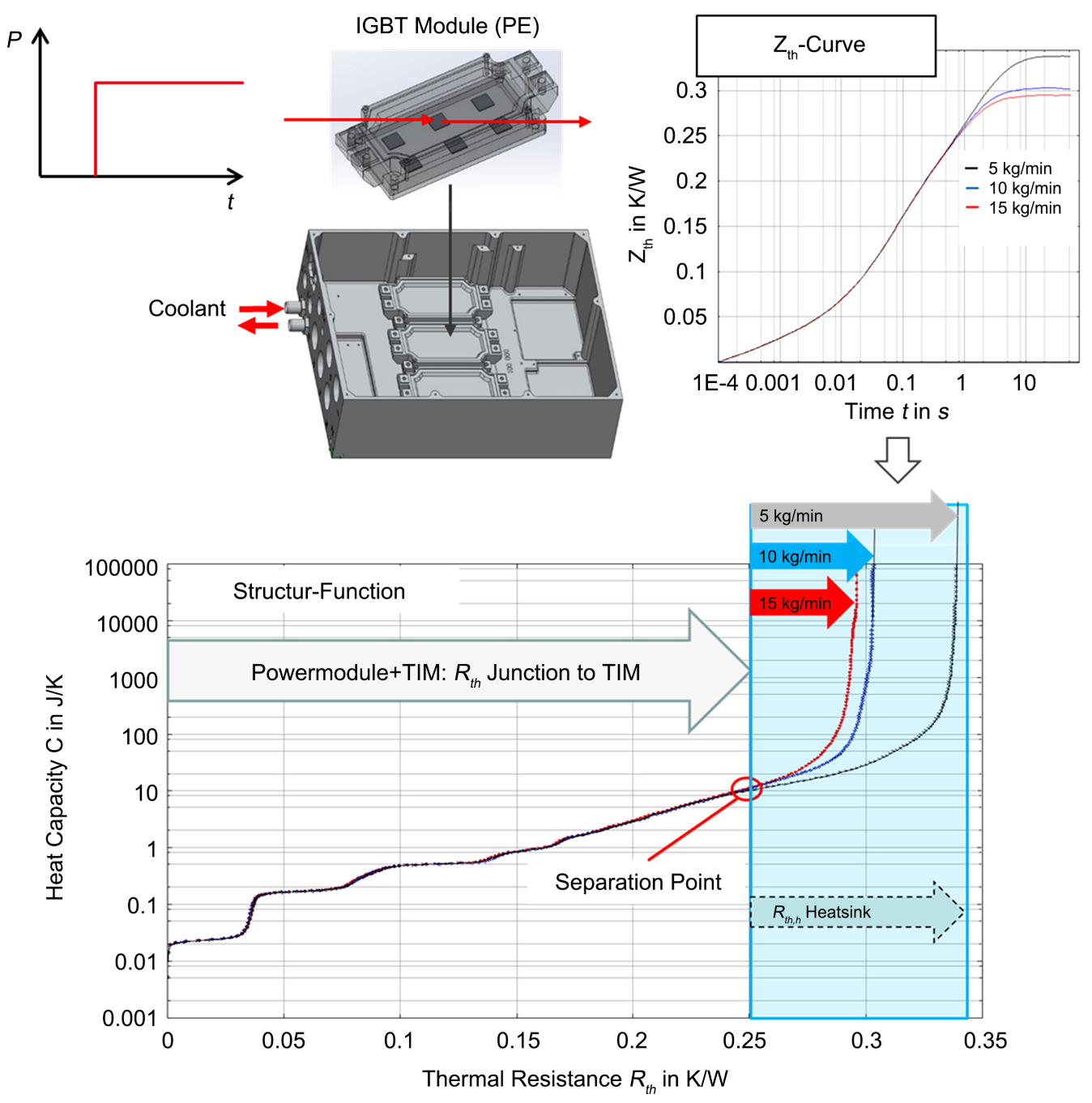

Figure 1. Measurement principle for the characterization of a heat sink in an oil circuit: $Z_{\text {th }}-$ Curves for mass flow $5 \mathrm{~kg} / \mathrm{min}, 10 \mathrm{~kg} / \mathrm{min}$ and $15 \mathrm{~kg} / \mathrm{min}$, in response to step heating of an IGBT-Module (top). Related structure function (bottom). 
The new measurement system is designed for temperatures ranging from $-40^{\circ} \mathrm{C}$ to $80^{\circ} \mathrm{C}$ and a mass flow of 0 to $20 \mathrm{~kg} /$ minute.

Figure 2(A) shows the entire setup for measuring the heat transfer coefficient and pressure drop. Figure 2(B) shows the enlarged view of the power electronics with chiller.

The power electronics (PE, IGBT) comprises three cells, which contains 6 semiconductors in each, and each semiconductor generates 25 watts of power. Total power generated from 3 cell PE units is 450 watts.

The main benefit of the new system as compared with conventional methods is that power electronics (such as IGBTs) and their cooling mechanism are accurately measured close to the application utilizing the original design, thereby establishing a solid basis for realizing optimal and cost-effective thermal management.

\section{Physical Properties of the Investigated Oils}

In the following, oils and water or water-glycol mixtures are compared concerning their heat transfer properties. Therefore, it is essential to discuss first the physical properties of these fluids, such as kinematic viscosity, specific heat, density, and electrical conductivity.
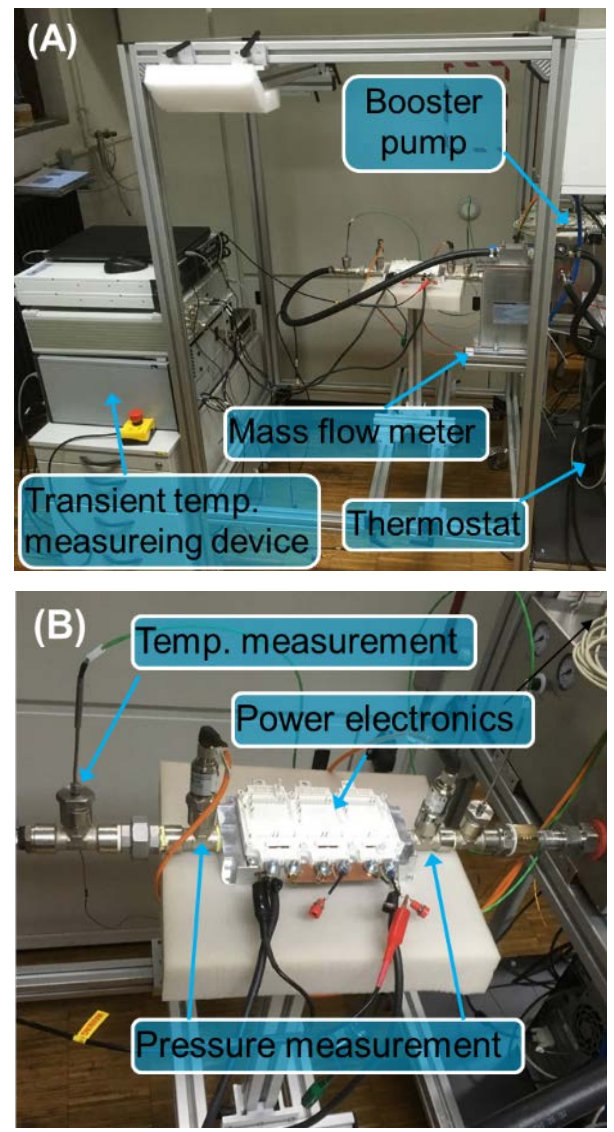

Figure 2. Setup for the measuring heat transfer coefficient and pressure drop (A) and enlarged view of the power electronics with chiller (B). 
Not only a base oil type, but also the viscosity as a result of the final fluid formulation mainly defines the thermal properties of the fluid [4] [5] [6]. Hence, a series of fluids were selected so that they cover a wide range of viscosity and base oil types. A commercially available additive package of a factory fill automatic transmission fluid (ATF) used by one of the major OEMs was treated in different base oil combinations as listed in Table 1. Various combinations of base oils, such as polyalphaolefin (PAO), gas-to-liquid (GTL), and ester, were used to vary viscosity. Often in lubricant applications, various base oils in different viscosities are mixed to obtain targeted viscosity. In this study, PAO 2, PAO 4, PAO 6, GTL 3 , and GTL 4 were used to achieve targeted viscosity at $100^{\circ} \mathrm{C}$ (A number after PAO or GTL denotes typical kinematic viscosity at $100^{\circ} \mathrm{C}$ in centistoke, cSt, or $\mathrm{mm}^{2} / \mathrm{s}$ ). In this work kinematic viscosities (KVs) at $100^{\circ} \mathrm{C}$ were varied from about $2.5 \mathrm{cSt}$ up to about $6.0 \mathrm{cSt}$ for finished fluids, see Table 1. Fluid JE is just a base oil mixture without additive package to understand additive effect on cooling performance. All other fluids, except JE, are using identical additive package with identical treat rate.

Cooling performance of all fluids can be easily understood by comparing the following combinations: Fluids JA, JB and JC as a function of viscosity; fluids JB and ATF as well as JC and JD as a function of base oil type; fluids JC, JD vs. JE as a function of additive package.

Ethylene glycol and ethylene glycol water mixtures were added as water-based coolant references. In these cases, additive package whose property is oil compatible and is water insoluble, was not incorporated as they are not compatible with each other.

Table 1 summarizes the relevant physical properties of the fluids at $75^{\circ} \mathrm{C}$.

Table 1. Physical properties of the fluids examined.

\begin{tabular}{|c|c|c|c|c|c|c|c|c|}
\hline \multirow{2}{*}{ Fluid ID } & \multirow{2}{*}{ BO composition } & \multirow{2}{*}{ Additive } & \multicolumn{5}{|c|}{$75^{\circ} \mathrm{C}$} & \multirow{2}{*}{$\frac{100^{\circ} \mathrm{C}}{v}$} \\
\hline & & & $k$ & $\rho$ & $c_{p}$ & $v$ & $\sigma @ 20 \mathrm{~Hz}$ & \\
\hline JA & $\mathrm{PAO} 4 / 6$ & Yes & 141.7 & 794.4 & 2.27 & 10.30 & 40 & 5.97 \\
\hline JB & $\mathrm{PAO} 2 / 4$ & Yes & 138.4 & 786.5 & 2.25 & 6.80 & 58 & 4.21 \\
\hline JC & $\mathrm{PAO} 2 / 4$ & Yes & 132.8 & 772.8 & 2.19 & 3.70 & 93 & 2.48 \\
\hline JD & PAO 2/Ester & Yes & 131.2 & 831.2 & 2.00 & 3.61 & 230 & 2.47 \\
\hline $\mathrm{JE}$ & $\mathrm{PAO} 2 / 4$ & No & 135.2 & 769.9 & 2.23 & 3.84 & 0.03 & 2.56 \\
\hline MR & NA & No & 422.5 & 1032.2 & 3.87 & 1.04 & $1.77 \mathrm{M}$ & NA \\
\hline EG & $\mathrm{NA}$ & No & 261.5 & 1066.5 & 2.73 & 3.09 & $0.17 \mathrm{M}$ & 1.94 \\
\hline ATF & GTL 3/4 & Yes & 142.1 & 783.6 & 2.28 & 6.55 & 64 & 4.10 \\
\hline Pure water & NA & No & 663.6 & 974.9 & 4.19 & 0.39 & 5500 & NA \\
\hline
\end{tabular}

With thermal conductivity $(\mathrm{k})[\mathrm{mW} /(\mathrm{m} \cdot \mathrm{K})]$, density $\left.(\rho) \mathrm{kg} / \mathrm{m}^{3}\right]$, specific heat capacity $\left(c_{p}\right)[\mathrm{J} /(\mathrm{g} \cdot \mathrm{K})]$, kinematic viscosity $(v)\left[\mathrm{mm}^{2} / \mathrm{s}\right.$ or $\left.\mathrm{cSt}\right]$, and electrical conductivity $(\sigma)[\mathrm{nS} / \mathrm{m}]$. 
Electrical conductivities $\sigma$ were significantly varied: there were about 8 orders of magnitude differences between only base oil mixture JE and ethylene gly$\mathrm{col} /$ water mixture MR. This is one of the important reasons why ethylene glycol-based cooling fluids (MR or EG) cannot be used for direct cooling of e-motor or power electronic units. Compared to EG or MR, additized fluid JD had EC of $230 \mathrm{nS} / \mathrm{m}$ at $75^{\circ} \mathrm{C}$, which is 4 orders of magnitude lower. The other additized fluids (JA, JB, JC and ATF) had even lower electrical conductivity compared to JD.

\section{Measurement Results}

Figure 3 shows as an example the structure functions of water/glycol 50/50 with mass flow between $2 \mathrm{~kg} / \mathrm{min}$ and $14 \mathrm{~kg} / \mathrm{min}$. The separation point marks the beginning of the heat transfer from the heatsink surface to the fluid.

The part of the structure functions (Figure 3 ) to the right of the separation point, provides the thermal resistance $R_{t h, h}=1 /(h \cdot A)$ from the heatsink to the adjacent fluid, with the heat transferring surface area of the power module $A=$ $330 \mathrm{~mm}^{2}$. The kink in the curves (pale blue mark above) comes from the thermostat control and plays no role in the evaluation.

Figure 4 summarizes the measured heat transfer coefficients of all fluids for a fluid flow rate from 6 to $14 \mathrm{~L} / \mathrm{min}$ and inlet temperature of $75^{\circ} \mathrm{C}$, according to the above equation. Please be aware that flow rate here is $\mathrm{L} / \mathrm{min}$, which is calculated from the actual measurement $(\mathrm{kg} / \mathrm{min})$ with density. Figure 5 shows only the oil-based fluids, which allows the scale of the $h$-axes to be reduced.

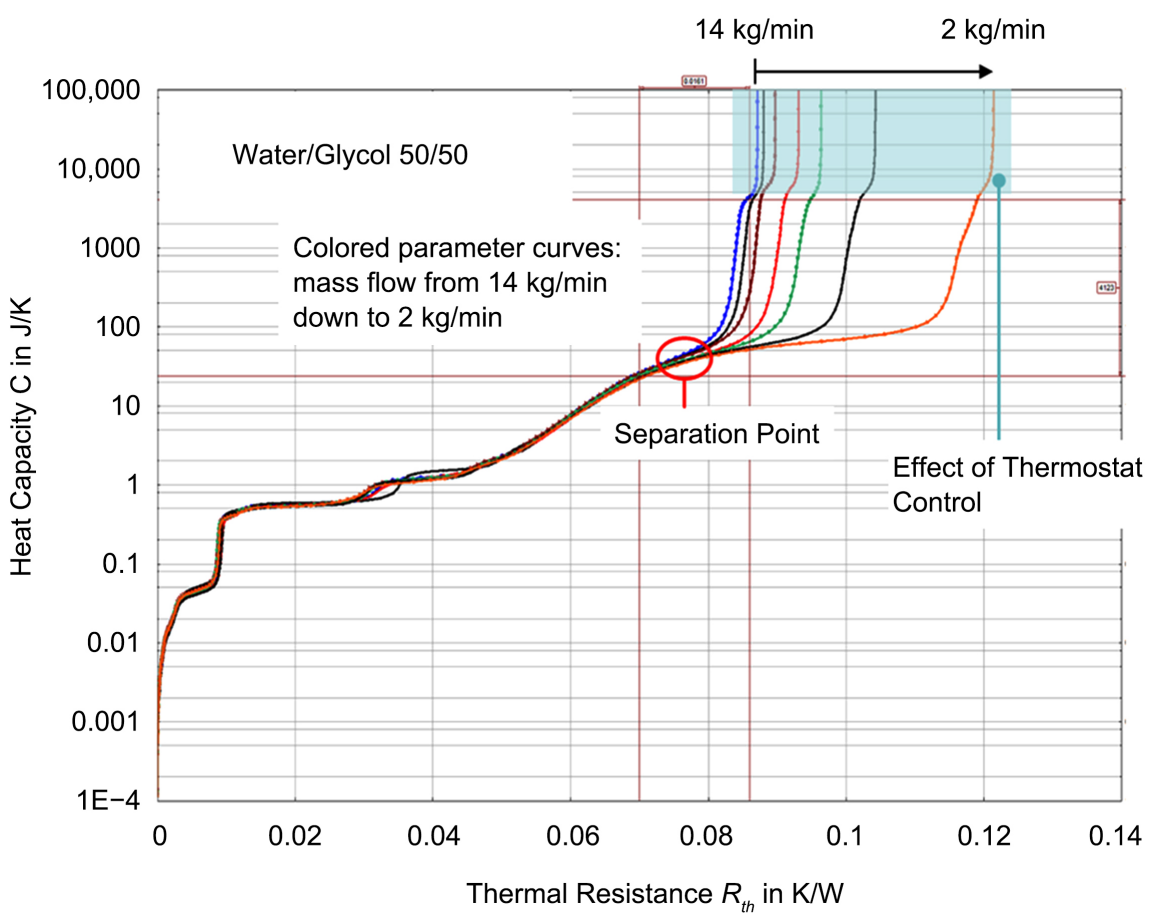

Figure 3. Structure functions of water/glycol 50/50 coolant with mass flow between 2 $\mathrm{kg} / \mathrm{min}$ and $14 \mathrm{~kg} / \mathrm{min}$. 


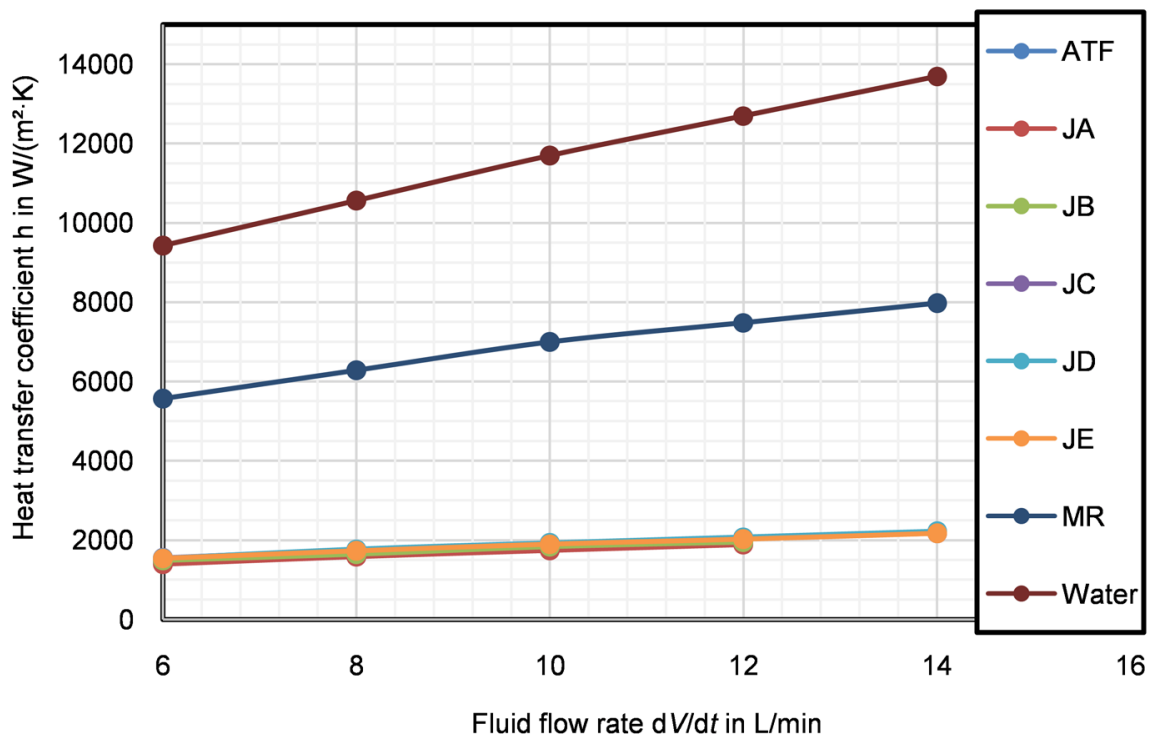

Figure 4. Heat transfer coefficients vs. flow rate, overview of all measured fluids.

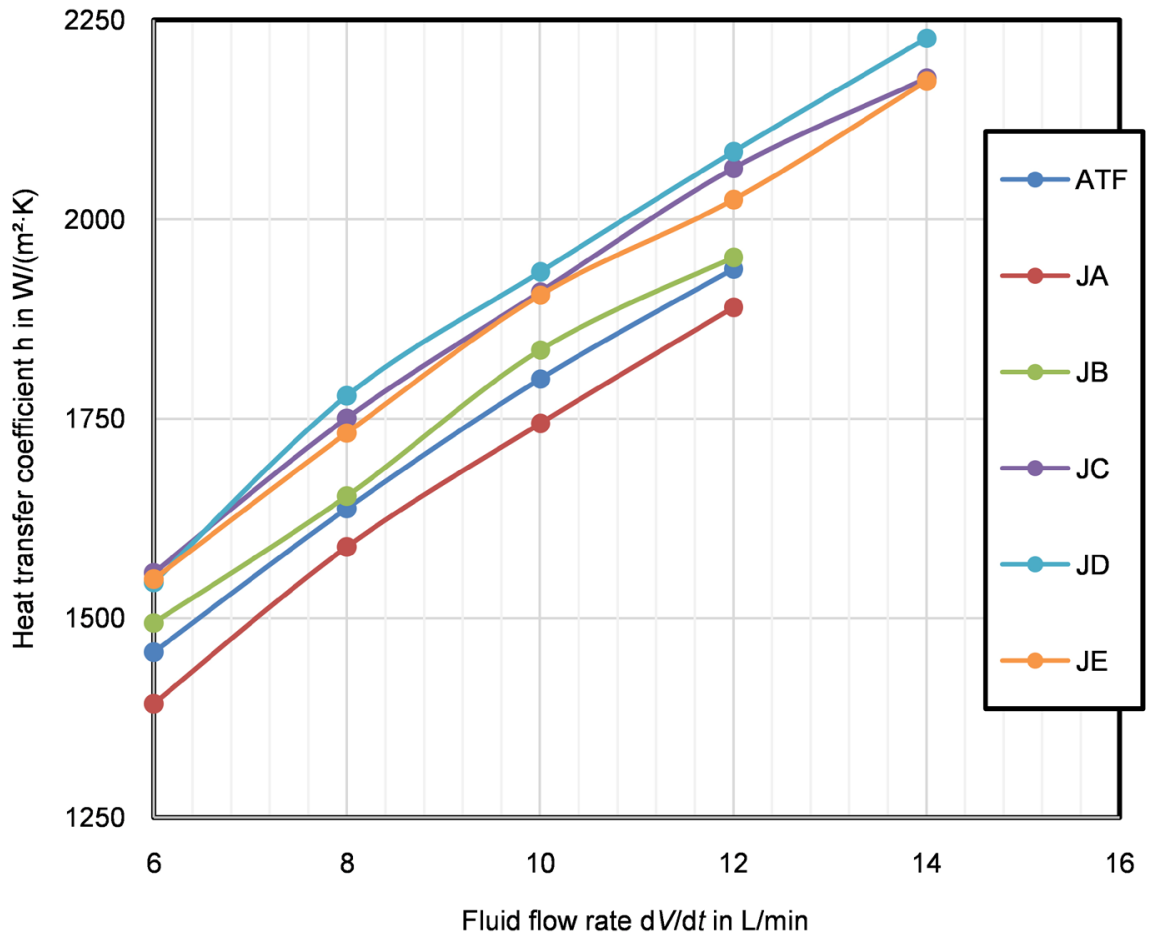

Figure 5. Heat transfer coefficients vs. flow rate, selection of oil-based fluids.

As can be seen in Figure 4 and Figure 5, $h$ values increase as the flow rate increases. Pure water has the highest $h$ value starting at $9400 \mathrm{~W} /\left(\mathrm{m}^{2} \cdot \mathrm{K}\right)$ at $6 \mathrm{~L} / \mathrm{min}$ flow rate and increases up to $13700 \mathrm{~W} / \mathrm{m}^{2} \cdot \mathrm{K}$ at $14 \mathrm{~L} / \mathrm{min}$ flow rate. The heat transfer coefficients $h$ of all other oil-based fluids were lowest around 1500 and $2170 \mathrm{~W} /\left(\mathrm{m}^{2} \cdot \mathrm{K}\right)$ at 6 and $14 \mathrm{~L} / \mathrm{min}$ flow rate, respectively. Water glycol mixture (MR) is placed around the middle between pure water and oil-based fluids. Pure water and water glycol mixture are about 6 and 3.5 times higher throughout the 
measured flow rate ranges, when compared to those of oil-based fluids. Taking a closer look at only oil-based fluids (Figure 5), JA revealed the lowest $h$ value with $1393 \mathrm{~W} /\left(\mathrm{m}^{2} \cdot \mathrm{K}\right)$ and JD revealed the highest value with $1550 \mathrm{~W} /\left(\mathrm{m}^{2} \cdot \mathrm{K}\right)$, both at $6 \mathrm{~L} / \mathrm{min}$ flow rate. Values of both fluids were trended very similarly at all other higher flow rates, with $1890 \mathrm{~W} /\left(\mathrm{m}^{2} \cdot \mathrm{K}\right)$ for $\mathrm{JA}$ and with $2085 \mathrm{~W} /\left(\mathrm{m}^{2} \cdot \mathrm{K}\right)$ for JD at $12 \mathrm{~L} / \mathrm{min}$ flow rate. Heat transfer coefficients $h$ values of the rest of the oil-based fluids are placed between these two, with commercial ATF being almost the center values. There was only $10 \%-11 \%$ differences between the lowest and highest $\mathrm{h}$ values, among oil-based fluids.

In addition to the heat transfer coefficient $h$, pressure drop $\Delta p$ across the heat sink was measured as a function of flow rate, Figure 6.

As expected, pressure differences of all fluids were increased as flow rate increased, and depended on fluids' physical properties, and in general oil-based fluids had high pressure drop compared to pure water. The trends for all fluids were very similar with only an exception of fluids MR and JD. MR had the highest pressure difference at lower flow rate up to about $9 \mathrm{~L} / \mathrm{min}$, but it ended up with lower value than JD at above $10 \mathrm{~L} / \mathrm{min}$ flow rate. Fluid JD, which includes ester base oil, showed higher resistance to flow as flow rate increased. This may be due to the structural difference of esters among other type of base oils studied here. Pure water had the lowest pressure differences as expected, due to the lowest viscosity.

\section{Discussion}

\subsection{Measuring Method}

The heat transfer coefficients $h$ (Figure 4, Figure 5) are calculated from the thermal resistance $R_{t h}=1 /(h \cdot A)$, which is the direct result of the measurement method. The area $A$ stands for the nominal heatsink surface, through which the heat flows $\left(A=330 \mathrm{~mm}^{2}\right)$. This consideration assumes that $A$ is isothermal, so the calculated $h$ is averaged over $A$. In reality, numerical simulation calculations show a few Kelvin of temperature difference on the surface, depending on the flow rate of the coolant.

The averaged $h$ corresponds to the value as it is important in practice as it stands for the total heat flow, which is transferred from the heat sink to the adjacent fluid.

Alternatively, it would be possible to measure the temperature at various points on the surface of the heatsink e.g. with thermocouples and to average it. However, this way of averaging the surface temperature is very time consuming and less accurate, since many thermocouples are necessary.

\subsection{Measurement Results}

As electrification of vehicle advances, OEMs envision a single fluid to perform well for all driveline applications, including e-motor, reduction gears, and PE units. 


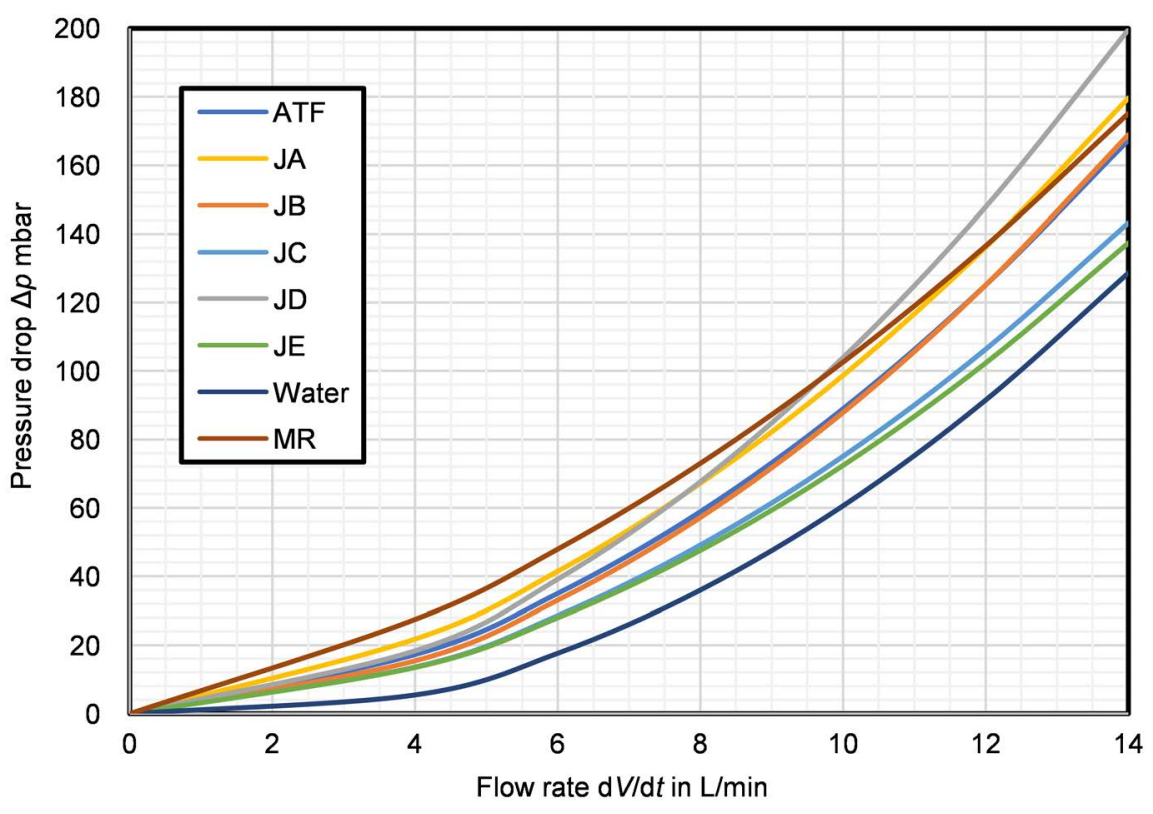

Figure 6. Pressure drop across the heat sink vs. flow rate.

Quantitative measurements of in-direct cooling performance of PE units through heat sink with pure water, water glycol mixtures, and various oil-based fluids, including commercial ATF, were performed in a newly developed measurement system.

Results indicated that heat transfer coefficients of oil-based fluids were extremely low compared to those of pure water or water glycol mixtures, which was about 3.5 to 6 times lower. On the other hand, electrical conductivity of pure water or water glycol mixtures were 3 - 5 orders of magnitude higher than typical oil-based fluids, and $7-8$ orders of magnitude higher than pure base oil, which is not a concern at all for in-direct cooling through the cooling channel but could be a major issue for direct cooling of PE units. Please be aware that higher electrical conductivity of a fluid is likely to interfere with the electrical signal PE units, which could lead malfunction of the PE units.

Within oil-based fluids, it was clear that viscosity and composition of base oils played significant roles for the heat transfer coefficient $h$ and pressure drop $\Delta p$. For example, fluids with lowest kinematic viscosity at $75^{\circ} \mathrm{C}$ (JC, JD and JE), which were about $3.7 \mathrm{cSt}$, provided higher heat transfer coefficient $h$ values compared to those with higher kinematic viscosity (JA), which was about 10.3 cSt (Table 1). Fluids with intermediate kinematic viscosity (JB and ATF) fell in between the previous two groups. The additive package did not play a major role in differentiating the heat transfer coefficient values, when compared with JC and JD versus JE. Another example was that the pressure drops of fluids JC and JE were lower than that of fluid JD, although their viscosities were almost the same. This is because the base oil structures in fluid JD, which includes diesters, are very different from the other two, which are PAO mixtures. Also, it was clear that water glycol mixtures had much higher pressure drops compared to pure 
water, and still had relatively higher pressure drops even compared to the rest of the oil-based fluids. Pressure drop was somewhat correlated to the viscosity of the fluids among oil-based fluids, except the ester-based fluid JD.

A simple comparison was made to roughly estimate the potential of direct oil cooling. For this, conventional cooling with water/glycol (50/50) in a circular tube was compared with direct oil spray cooling. Table 2 contains the assumed physical properties of the compared fluids.

The tube diameter of the indirect water/glycol cooling was assumed to be 20 $\mathrm{mm}$. In case of oil cooling, the heat transfer coefficient was calculated for a nozzle with a $5 \mathrm{~mm}$ round opening, $25 \mathrm{~mm}$ distance to the sample and an oil spray radius on the sample of $25 \mathrm{~mm}$. Figure 7 shows the calculated values as a function of fluid flow.

The curves (Figure 7) were calculated with equations from Schlünder and Gnielinski (spray cooling [7]) and VDI Wärmeatlas (turbulent tube flow [8]). The values in direct spray cooling with oil are around 3 to 8 times higher than in indirect cooling with water/glycol.

Of course, this rough comparison can only show one aspect of direct oil cooling. For a comprehensive comparison between direct oil cooling and indirect water/glycol cooling, one would also have to consider the total heat path from the heat source to the heat sink, the cooled area, the technical infrastructure and questions of lifetime.

Table 2. Physical properties of the fluids for the comparison of indirect verses direct oil cooling concept.

\begin{tabular}{ccc}
\hline & Water/glycol & Oil \\
\hline$k(\mathrm{~mW} /(\mathrm{m} \cdot \mathrm{K}))$ & 423 & 130 \\
$v\left(\mathrm{~mm}^{2} / \mathrm{s}\right.$ or cSt $)$ at $75^{\circ} \mathrm{C}$ & 1.0 & 3.7 \\
Prandtl number & 10 & 80 \\
\hline
\end{tabular}

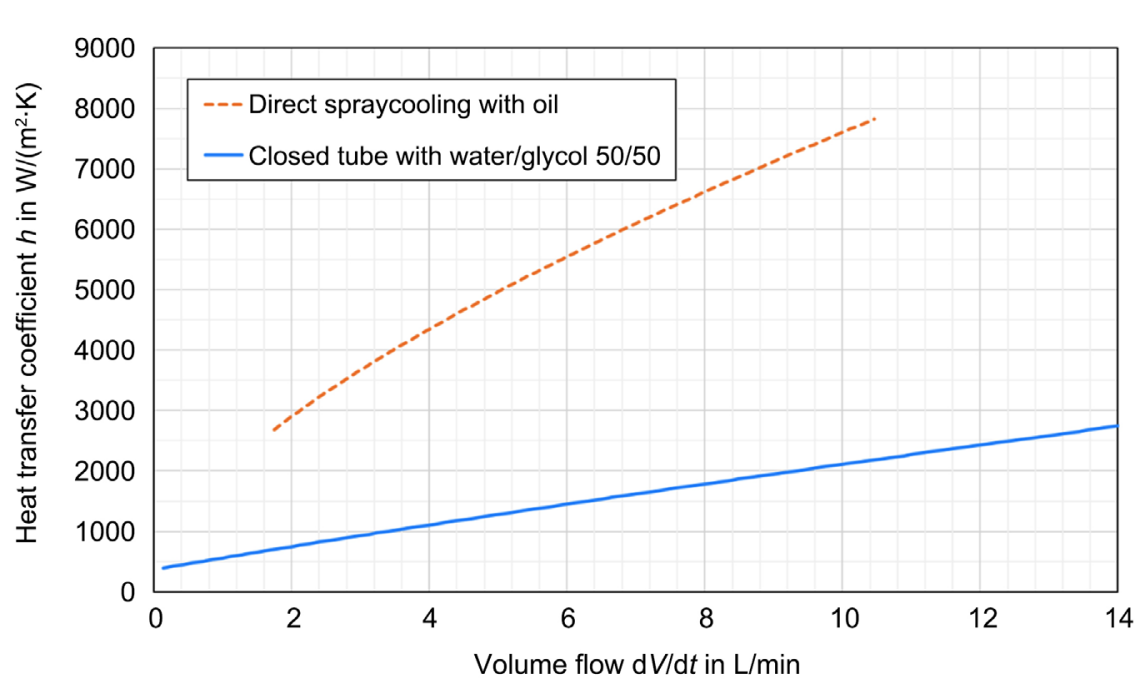

Figure 7. Comparison of calculated heat transfer coefficients of direct oil spray cooling and water/glycol (50/50) tube cooling as a function of volume flow. 


\section{Conclusions}

A conventional cooling system of the PE includes a cooling jacket that cools PE units indirectly using water/glycol as a cooling media. The continuously increasing heat losses in the PE modules require a more efficient thermal management. In general, this can be achieved by improving the thermal conductivity of the cooling media or by improving the heat transfer between the cooling media and the source of the heat generation. The property of heat transfer is dependent on the hardware design and measurement of it is a nontrivial task.

For this purpose, ZFW developed a dedicated tool where PE modules are utilized to generate defined heat energy and detect the thermal response at the same time. The application of the thermal transient method allowed the authors to derive a heat transfer coefficient, which represents the combinations of the heat transfer of a fluid as well as design-dependent heat transfer between the surface and the cooling medium. Both water/glycol-based cooling media and transmission fluids have been investigated for heat transfer coefficient in the new setup. Furthermore, the gained knowledge was transferred to the direct cooling concepts which are currently under discussion in the industry. It was found that direct oil cooling with transmission fluids could increase heat transfer coefficient by a factor of 3 to 8 , compared to the indirect cooing with water/glycol as cooling media.

In conventional cooling systems, due to their physical properties, oil-based fluids are less suitable as a cooling medium than water/glycol mixtures. However, in the case of direct cooling, oil-based fluids could have decisive advantages due to their low electrical conductivity. This requires further investigations, such as optimization of the cooling structure in the flow channel to minimize pressure drop and the cooling medium parameters i.e., thermal conductivity, viscosity, and density. Nonetheless, the direct oil cooling concept would enhance the application of transmission fluids in vehicle electrification and promise significantly improved thermal management.

\section{Conflicts of Interest}

The authors declare no conflicts of interest regarding the publication of this paper.

\section{References}

[1] JEDEC STANDARD JESD51-14 (2010) Transient Dual Interface Method for the Measurement of the Thermal Resistance Junction to Case of Semiconductor Devices with Heat Flow Trough a Single Path. JEDEC Solid State Technology Association, Arlington.

[2] Griesinger, A. (2019) Wärmemanagement in der Elektronik, Theorie und Praxis, Springer Vieweg. Springer-Verlag GmbH Deutschland, Heidelberg. https://doi.org/10.1007/978-3-662-58682-2

[3] Bennion, K. and Moreno, G. (2015) Convective Heat Transfer Coefficients of Automatic Transmission Fluid Jets with Implications for Electric Machine Thermal 
Management, National Renewable Energy Laboratory. International Technical Conference and Exhibition on Packaging and Integration of Electronic and Photonic Microsystems (InterPACK), San Francisco, 6-9 July 2015.

[4] Kwak, Y., Cleveland, C., Adhvaryu, A., Fang, X., Hurley, S. and Adachi, T. (2019) Understanding Base Oils and Lubricants for Electric Drivetrain Applications. SAE International, 2019-01-2337.

https://doi.org/10.4271/2019-01-2337

[5] Beyer, M., et al. (2019) Lubricant Concepts for Electrified Vehicle Transmissions and Axles. Tribology Online, 14, 428-437.

[6] Maelger, H., Petuchow, A., Plaatje, A., Yungwan K. and Banks A. (2019) Realistic Testing to Assess How Electrified Transmission Fluids Will Withstand Ageing, CTI Symposium. 18th International Congress and Expo, Berlin, 10-11 December 2019.

[7] Schlünder, E.U. and Gnielinski, V. (1967) Wärme- und Stoffübertragung zwischen Gut und aufprallendem Düsenstrahl. Chemie Ingenieur Technik, 39, 578-584. https://doi.org/10.1002/cite.330390915

[8] VDI-Wärmeatlas (2013) 11. Auflage. Springer, Berlin, 789. 